\title{
Point of technique
}

\section{The optimum radiographic follow-up of a giant vertebrobasilar junction aneurysm which treated with endovascular coil embolization}

\author{
${ }^{1}$ L.C. Şahika ${ }^{2}$ E. ̈Ü Mehmet and ${ }^{3}$ S. Karaköse \\ 1,2 Department of Neurosurgery and 3 Department of Radiology, Selcuk University, Meram Faculty of \\ Medicine, Konya \\ Reprints to Dr ŞAHIKA LiVA CENGIZ Department of Neurosurgery A/5, , Meram Faculty of Medicine, \\ Selcuk University Akyokuş 42080 /Konya/ Turkey \\ e-mail: livacengiz@yahoo.com
}

\section{Introduction.}

In our a study a giant wide-necked vertebrobasilar junction aneurysms previously treated with endovascular detachable coils is followed up meticulously. The outcome was satisfactory

A 52 year old man presented with 2 year history of headache and was diagnosed as partially thrombosed giant aneurysm at the vertebrobasilar junction on cerebral angiographic examination. The patient underwent coil embolization by inserting a detachable and hydrogel coated coils successfully. He became symptom free after embolization on the next day. But 16 months later he was readmitted with a reccurrent episode of headache. Subsequent cerebral angiography revealed a new baby aneurysm formation at the same level near by the neck of the previous aneurysm.

Although giant vertebrobasilar junction aneurysm seems to be successfully blocked off initially, it should be remembered long- term follow-up as 1,5-2 years is required to further validate the importance of coil embolization technique.

Key Words: Endovascular coil embolization, long term follow up, vertebro basilar junction aneurysm.

Case Report

A 52 year old man presenting with 2 years headache, was admitted to a neurology department of a peripheral hospital . Computed tomography of the skull demonstrated a hemorrhage suggesting a thrombosed basilar trunk aneurysm. The patient was transferred to our department for further work-up and management. On admission to our unit, neurological examination revealed right central facial paralysis, speech disturbances, $2 / 5$ right hemiparesis, bilaterally right nystagmus and physical examination was negative. A partially thrombosed aneurysm was spotted at the basilar artery trunk and an aneurysm $25 \times 15 \mathrm{~mm}$ in diameter was located at the vertebrobasilar junction on cranial tomography and CT angiography, respectively (Fig 1,2)

Via a transfemoral selective right vertebral artery angiography, a giant vertebrobasilar junction saccular aneurysm was detected just proximal to the basilar artery. The diameter of the neck was $4 \mathrm{~mm}$, and dimension of the aneurysm was $35 \mathrm{~mm} \times 15 \mathrm{~mm}$ at the junction of vertebral and basilar artery bifurcation (Fig 3). The patient underwent coil embolization by inserting a detachable hydrogel coated coil. The immediate postoperative angiography showed successful occlusion of aneurysmal sac with preservation of basilar artery (Fig 4). The patient was discharged from hospital with improved right hemiparesis $(3 / 5)$ with no more headaches. However 16 months later the patient re-presented to our department with progressive headache after apparent good health since the initial embolization. Subsequent angiography revealed a new aneurysm had formed near the neck of the previous aneurysm(Fig 5,6) and had started to be symptomatic 
Fig1/2

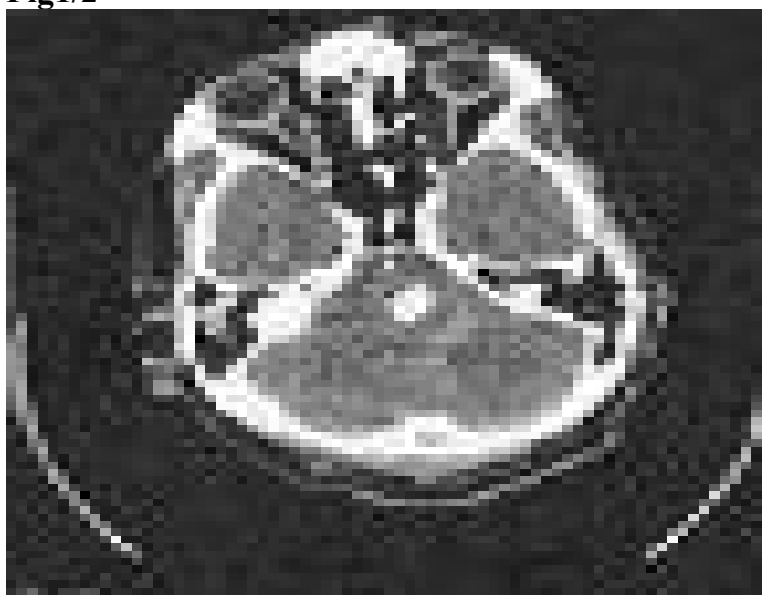

fig 3/4

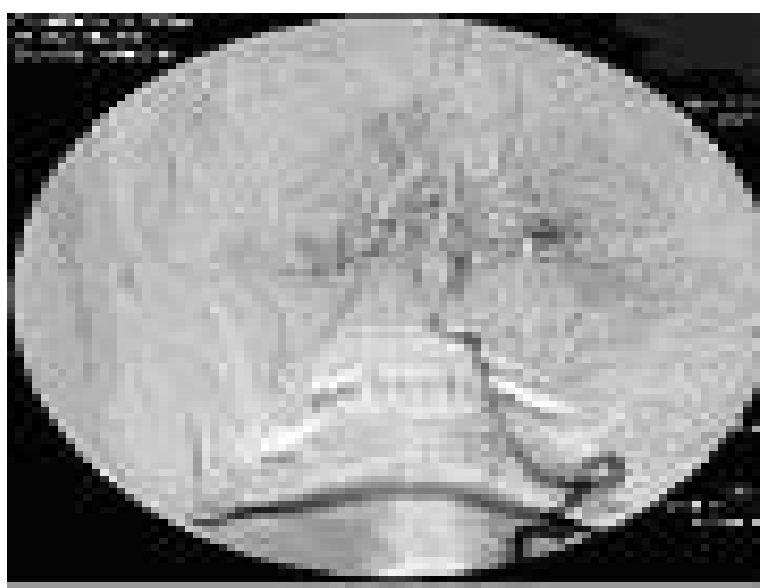

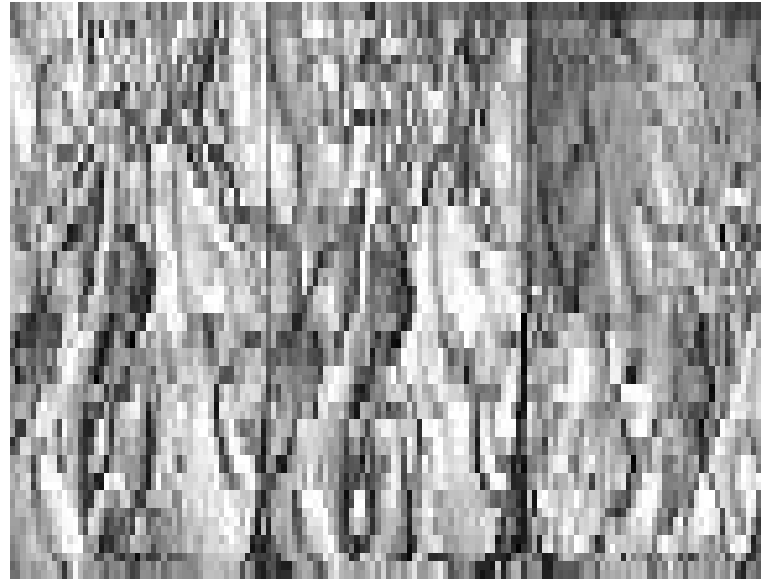

fig4
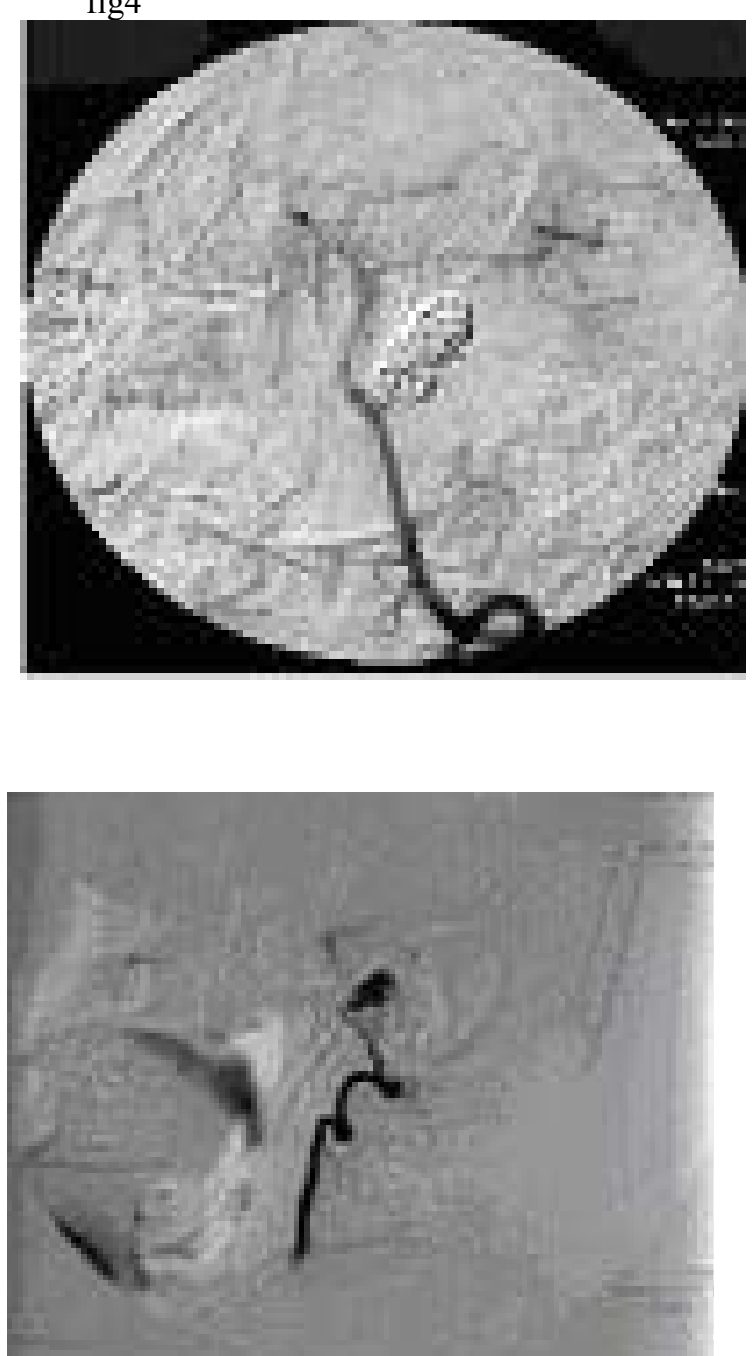

Fig $5 / 6$

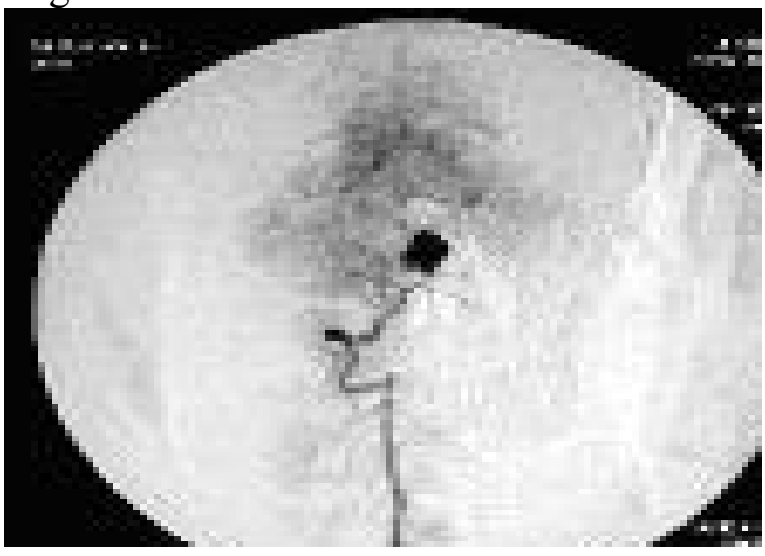

FIG 1: A thrombosed aneurysm appearance at the basilar artery trunk on cranial tomography.
FIG 2: An aneurysm in $25 \times 15 \mathrm{~mm}$ diameters located vertebrobasilar junction were observed on CT angiography. 
FIG 3: On transfemoral selective right vertebral artery angiography a vertebrobasilar junction saccular giant aneurysm was detected just proximally to basilar artery. The diameter of the neck was $4 \mathrm{~mm}$, and dimension of the aneurysm was $35 \mathrm{~mm} \times 15 \mathrm{~mm}$. at the junction or vertebral and basilar artery bifurcation

FIG 4: The cerebral angiography after coil embolization by inserting a detachable and hydrogel

\section{Discussion}

Aneurysms arising at or near the bifurcation of the basilar artery are some of the most difficult aneurysms encountered in the practice of the cerebrovascular surgery. The complex anatomy of the posterior fossa, the highly sophisticated neural content and the depth of the operative field make these lesions particularly difficult ${ }^{1}$. Endovascular treatment of wide necked aneurysms by using two microcatheters techniques may be feasible and safe ${ }^{2}$. Endovascular occlusion of these aneurysms also decreases the possibility of unwanted occlusion of perforating arteries to the brainstem ${ }^{2}$. Guglielmi detachable coil (GDC) procedure is a safe and reliable means of preventing aneurysm hemorrhage in patients with small and large intracranial aneurysms but are less satisfactory involving giant lesions ${ }^{3}$. The diameter of the aneurysm larger than $25 \mathrm{~mm}$ are called giant aneurysms and 50-60 percent of giant aneurysms are thrombosed as it is the case in our patient. ${ }^{4}$. It is to be emphasized that vertebrobasilar junction aneurysms are frequently associated with fenestrations and can be successfully treated with GDCs ${ }^{6}$. In such cases CT angiography may be an additional valuable tool for better understanding of complex anatomy of aneurysms associated with fenestration ${ }^{6}$ Postcarotid endarterectomy pseudoaneurysms are rare complications and can also be treated with combined stent graft and coil embolization 1. In our patient fenestration of vertebrobasilar junction was not detected and no new bleeding occurred during follow-up period. The wide necked intracranial artery aneurysms was treated using a combination of endovascular stent implantation across the aneurysm neck and

\section{References}

1. Seruga T, Klein GE .Endovascular treatment of intracranial artery aneurysms in the posterior cerebral circulation. Wien Klin Wochenschr. 2004;116 Suppl 2:13-8.

2. Kwon OK, Kim SH, Kwon BJ, et all. Endovascular treatment of wide-necked aneurysms by using two microcatheters: techniques and outcomes in 25 patients.AJNR Am J Neuroradiol. 2005 ;26(4):894-900.

3. Malisch TW, Guglielmi G, Vinuela F, Duckwiler G, Gobin YP, Martin NA, et all.. Intracranial aneurysms treated with the Guglielmi detachable coil: midterm clinical results in a consecutive series of 100 patients. J Neurosurg. 1997 coated coils showed successful occlusion of aneurismal sac with preservation of basilar artery .

FIG 5: The AP appearance of new aneurysm near by the neck of the ancient aneurysm. 5 months later on cerebral angiography .

FIG 6: The lateral appearance of a new aneurysm near by the neck of the ancient aneurysm 5 months later.

endovascular coil placement to obliterate the aneurysm provides satisfactory outcome ${ }^{5}$. In our patient stent implantation was not suitable due to anatomic narrow structure of the right vertebral artery, so coil obliteration was performed. Embolization related mortality is quoted as 3.33\%, permanent neurological deficit as $1.67 \%$ and the transitory neurological deficit as $3.33 \%{ }^{1}$. Follow-up angiograms may show recanalization caused by coil impaction, remnants, thrombosis, rupture or rebleeding of aneurysm, over embolization , protrusion of coil into parent artery ${ }^{3}$. Although the surgically difficult aneurysms such as vertebrobasilar junction aneurysms seem to be successfully treated initially with GDCs, the complications such as new aneurysms may emerge in the course of time. The follow up angiogram revealed the new baby aneurysm formation at the same level of the old aneurysm 16 months from treating the first one. Thus, we thought an optimum follow-up time should be from 1,5 to 2 years, and further long- term followup time is required contrary to the mean radiographic follow-up period of 11 months in literature ${ }^{7}$ Consequently we conclude that skilled embolization technique, efficient angio-anatomy of an aneurysm and its parent artery, correct judgment of embolic materials, and long angiographic follow up interval are beneficial in the early detection,treatment and reduction of the gravity of these complications.Acknowledgment: This procedure was carried out by Professor at Radiology Department, Selcuk University, Meram Faculty of Medicine, Konya

Aug;87(2):176-83. Erratum in: J Neurosurg 1998 ;88(2):359.

4. Casasco A, Arnaud O, Gobin P,et all. Giant intracranial aneurysm. Elective endovascular treatment using metallic coils. Neurochirurgie. 1992;38(1):18-26.

5. Islak C, Kantarci F.Vertebrobasilar junction aneurysms associated fenestration: experience of five cases treated with Guglielmi detachable coils. Surg Neurol 2004;61:248-254.Surg Neurol. 2004;62(5):470; author reply 470-471.

6. Chow MM, Thorell WE, Rasmussen PA. Aneurysm regression after coil embolization of a 
concurrent aneurysm.AJNR Am J Neuroradiol. 2005 ;26(4):917-921.

7. Hoh BL, Carter BS, Putman CM, Ogilvy CS Important factors for a combined neurovascular team to consider in selecting a treatment modality for patients with previously clipped residual and recurrent intracranial aneurysms.

Neurosurgery2003;52(4):732-738 


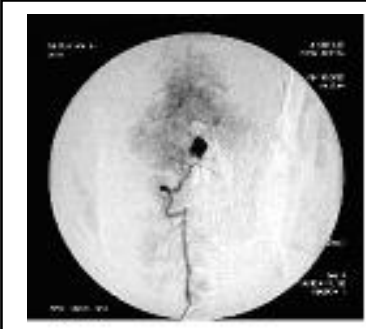

um radiographic follow up of a giant vertebro-basilar junction aneurysm Sahika LC et al

FIGURE 5 


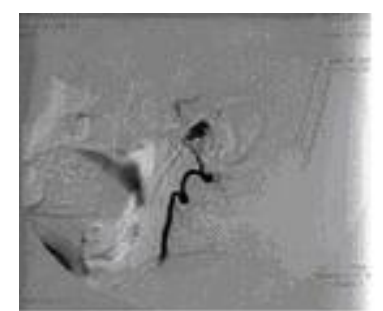

FICURE 6 